\title{
Safe Platooning in the Event of Communication Loss using the Flatbed Tow Truck Model
}

\author{
Alan ALI \\ Institut de Recherche en Communications \\ et Cybernétique de Nantes (IRCCYN) \\ Ecole Centrale de Nantes (ECN) \\ Nantes, France \\ Email: Alan.Ali@irccyn.ec-nantes.fr
}

\author{
Gaëtan GARCIA \\ Ecole Centrale de Nantes (ECN) \\ Nantes, France \\ Email: Gaetan.Garcia@ec-nantes.fr
}

\author{
Philippe MARTINET \\ Institut de Recherche en Communications \\ et Cybernétique de Nantes (IRCCYN) \\ Ecole Centrale de Nantes (ECN) \\ Nantes, France \\ Email: Philippe.Martinet@irccyn.ec-nantes.fr
}

\begin{abstract}
Optimizing inter-vehicle distances is very important to reduce traffic congestion on highways. A modification of the constant time headway policy (CTH) has been proposed in [1]. This modification significantly reduces inter-vehicle distances, but this reduction in the inter-distance may increase the risks of collisions. In this paper, the safety of the modified CTH applied to a homogeneous platoon is addressed. Many critical scenarios are discussed, including hard braking of the leader and followers in the event of communication loss. Safety conditions are presented. In addition, a method to find the maximum allowed delay to inform all the vehicles about communication loss, is also presented. Simulations have been run with 10 vehicles to check safety in the proposed scenarios.
\end{abstract}

\section{INTRODUCTION}

Nowadays, traffic congestion, pollution, and people safety problems become more and more important due to the explosion in the number of cars.

Driving in platoon has many advantages. It increases traffic density and security, and at the same time it decreases fuel consumption and driver tiredness [12].

Many platoon models and control laws can be found in [14]. Some of the proposed models do not require inter-vehicle communications, while others may require full communications. Other authors have modeled platoons using physics-inspired models [17], mimicking animal interaction behaviors or using multi-agent systems model [8].

The stability of a platoon is defined by String stability [14], [11]. String stability means that errors do not amplify as they propagate through the platoon, and that errors have the same sign to avoid collisions.

In addition to string stability, safety must be ensured. Th platoon is safe if it is guaranteed that no vehicle (or platoon) will collide with the vehicle (or platoon) a head of it at a relative speed greater than a prescribed limit [5]. In the literature, there are two ways to ensure safety: 1- by imposing some constraints on the control law and its parameters [13], [10], [6], [5], [4], 2- using supervisors to observe the state of the system and manage the switch between low level controllers [7]. The first method usually leads to simpler systems.

In this work, we concentrate on the longitudinal control of platoons on highways. We study the safety of the homogeneous platoon when using the modified CTH law proposed in [1].
Critical scenarios are discussed. These scenarios include leader and followers hard braking simultaneous to communication loss. The delay of informing all the vehicles about communication loss is also addressed and the maximum value of this delay, without losing safety, is determined.

The paper is organized as follows. Section II describes the vehicle and platoon models. The control is presented in section III. Section IV introduces safety and critical scenarios. In section V we present the simulations. Finally, in section VI conclusions and perspectives are presented.

\section{Modeling}

In the case of platooning on highways, where the road curvature is small, it is well known that longitudinal and lateral controls can be considered as decoupled. In this paper, we also make this safe assumption, and we study only the longitudinal control.

\section{A. Longitudinal Dynamic Model Of The Vehicle}

By using Newton's law and applying exact linearization, we get the following second order linear system [16], [1] :

$$
\ddot{x}=\dot{v}=W
$$

where $x$ is the position of the vehicle, $v$ the speed of the vehicle and $W$ is the control input for the linearized system.

\section{B. Platoon Model}

The platoon is a set of vehicles following each other, running at the same speed and keeping a desired distance $L$ between two consecutive vehicles, as shown in fig. 1.

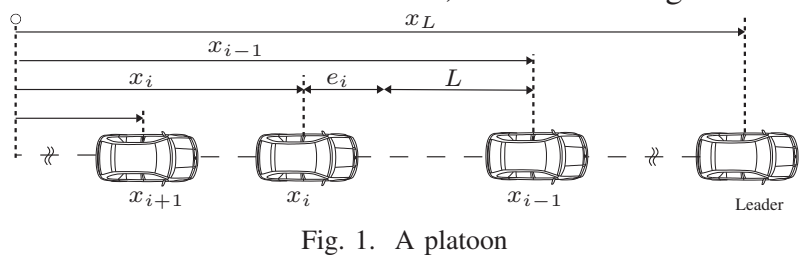

Assuming a point mass model for all the vehicles, we define the spacing error of the $i$ th vehicle:

$$
e_{i}=\Delta x-L=x_{i-1}-x_{i}-L
$$


where $x_{i}$ is the position of the $i$ th vehicle, $\Delta x$ the spacing between the $i$ th vehicle and the $(i-1)$ th vehicle, and $L$ the desired inter-vehicle distance.

The platoon model shown in fig. 2 is called flatbed tow truck model [3]. In this model, the vehicles in the platoon are carried on a virtual truck which moves at a speed $V$, and the $i$ th vehicle moves with a speed $v_{i}-V$ relative to the truck.

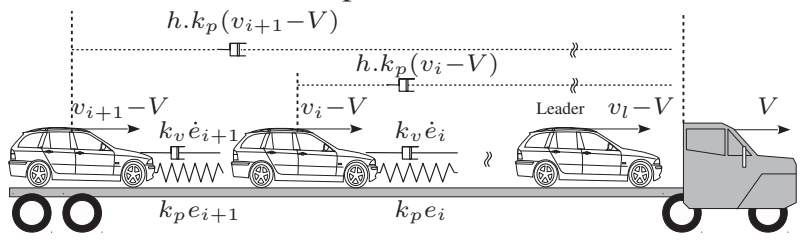

Fig. 2. Flatbed tow truck model

\section{Platoon CONTROL With AND Without COMMUNICATIONS}

\section{A. Control Law}

We have proposed in [1], [2], [3] a modification of the classical CTH law. This modification allowed to reduce the inter-vehicle distances. required to ensure string stability of the platoon, making them nearly equal to the distances in the case of the constant distance policy.

We have proposed the following control law :

$$
W_{i}=\frac{1}{h} \dot{e}_{i}+\frac{\lambda}{h} e_{i}-\lambda\left(v_{i}-V\right)
$$

This control law make the platoon stable with the following transfer function of the spacing error:

$$
G_{i}(s)=\frac{e_{i}(s)}{e_{i-1}(s)}=\frac{1}{h s+1}
$$

This control law was deeply studied in [1], [2], [3]. String stability of the platoon was proved. Robustness to actuation lags was also studied and the condition of stability in presence of lags was presented. The effectiveness of the control law even in case of total communication loss has also been proved, but the safety of the platoon was not discussed.

\section{B. Control In The Event Of Communication Loss}

Introducing the parameter $V$ in the modified CTH implies inter-vehicle communication. In [1], [3], we proved that the only condition on the value of $V$, to ensure string stability, is to make $V$ identical for all vehicles at any sample time. We also proved that stability can be preserved even if communication is totally lost, by switching to the classical time headway policy $(V=0)$ and using only on board information [9].

Switching to classical mode must be smooth to prevent undesirable response. This can be achieved by decreasing the value of $V$ smoothly. When the communication is lost, each vehicle must decrease $V$, independently from other vehicles, using the same predefined decreasing rate. This ensure that string stability is preserved. We will discuss later how to set the value of this rate.

\section{SAFETY}

\section{A. Safe Control}

The unsafe impact definition is given in [4] and it is said to happen at time $t$ between $i$ th vehicle and $(i-1)$ th vehicle if

$$
\Delta x \leq 0 \quad \text { and } \quad-\Delta \dot{x} \geq v_{\text {allowed }}
$$

Where $v_{\text {allowed }}$ is the maximum allowable impact speed.

We define the set $X_{M S} \subset \Re^{3}$ as the set of triples $\left(\Delta x, \Delta \dot{x}, v_{L}\right)$ which do not satisfy condition (5).

A control law $W$ for the trail vehicles is said to be safe for an initial condition $\left(\Delta x(0), \Delta \dot{x}(0), v_{L}(0)\right)$ if for all $t \geq 0$ it satisfy the following [5] :

$$
\left(\Delta x, \Delta \dot{x}, v_{L}\right) \in X_{M S}, \quad\left\{\begin{array}{l}
\forall a_{L} \in\left[a_{\min }, a_{\max }\right] \\
0 \leq v_{L} \leq v_{\max }
\end{array}\right\}
$$

where $a_{L}$ is leader's acceleration, $a_{\max }$ is the maximum leader acceleration, $a_{\min }$ is the maximum leader deceleration, $v_{L}$ is the speed of the leader, $v_{\max }$ is the maximum speed.

\section{B. Hard Braking Of The Leader}

One method to check the safety of the platoon is to consider a hard braking scenario. In this scenario, the leader vehicle applies an emergency stop with its maximum deceleration and the other vehicles in the platoon brake according to their control law [15].

In the following, we prove that the spacing between the leader and the first follower $\Delta x_{1}$ is the minimum inter-vehicle spacing in the platoon in case of any disturbance in $\Delta x_{1}$. Then, we find the conditions on the control law which maintain this minimum distance always greater than zero, so we ensure safety.

In [1] we have proved string stability of the platoon, when using the control law given in (3), using a sufficient string stability condition found in [11]:

$$
\left\|e_{1}\right\|_{\infty} \leq \ldots \leq\left\|e_{i}\right\|_{\infty} \leq\left\|e_{i-1}\right\|_{\infty}
$$

So in the case of any spacing disturbance between the leader and the first follower, the maximum amplitude of the first spacing error $\left\|e_{1}\right\|_{\infty}$ will be the largest spacing error in the platoon. So bounding $\left\|e_{1}\right\|_{\infty}$ in normal and leader's hard braking scenarios ensures safety of the whole platoon.

Choosing $v_{L}$ as the shared speed $V$ and using eq. (3) and (1), in addition to $a_{L}=\ddot{x}_{L}$, where $x_{L}$ is the position of the leader, we get the dynamics of the first error in the platoon:

$$
h \ddot{e}_{1}+(1+\lambda h) \dot{e}_{1}+\lambda e_{1}=h a_{L}
$$

We calculate the transfer function:

$$
G_{1}(s)=\frac{e_{1}(s)}{a_{L}(s)}=\frac{h}{h s^{2}+(1+h \lambda) s+\lambda}
$$

In this case the impulse function is $g_{1}(t)$.

So $e_{1}$ is defined by the acceleration of the leader. From [14] we get $\|G(\omega)\|_{\infty}=\|g(t)\|_{1}$ when we choose $g_{1}(t)>0$. Hence, the maximum value of $e_{1}(t)$ can be bounded by the following relation: 


$$
\left|e_{1}(t)\right| \leq\left\|G_{1}(\omega)\right\|_{\infty}\left\|a_{L}(t)\right\|_{\infty}
$$

Keeping $\left|e_{1}(t)\right|<L_{e}<L$ ensures that $\Delta x>0$, so according to definition (5) no unsafe impact takes place. $L_{e}$ will be the maximum limit for spacing error using the modified CTH. $L-L_{e}$ is the safety distance margin. In this case, the following inequality is a sufficient condition to ensure safety:

$$
\left|e_{1}(t)\right| \leq\left\|G_{1}(\omega)\right\|_{\infty} \max \left(\left|a_{\max }\right|,\left|a_{\min }\right|\right) \leq L_{e}
$$

so we get:

$$
\omega^{4}+\left((1+\lambda h)^{2}-2 \lambda\right) \omega^{2}+\lambda^{2}-\frac{h^{2} a_{\min }^{2}}{L_{e}^{2}} \geq 0 \quad \forall \omega
$$

The above inequality holds if one of the following sufficient conditions is true:

1) The $2^{\text {nd }}$ degree polynomial in $\omega^{2}$ has no root (the discriminant is negative) and $\lambda \geq \frac{h\left|a_{\min }\right|}{L_{e}}$.

2) The coefficients of the polynomial are both positive.

This gives us the following sufficient conditions for safety:

$$
\left\{\begin{array}{l}
\lambda \geq \frac{h\left|a_{\min }\right|}{L_{e}} \\
\eta^{2}-2 \lambda>0
\end{array}\right\} \text { or }\left\{\begin{array}{l}
\lambda \geq \frac{h\left|a_{\min }\right|}{L_{e}} \\
\eta^{4}+\frac{4 h^{2} a_{\min }^{2}}{L_{e}^{2}}<4 \lambda \eta^{2}
\end{array}\right\}
$$

where $\eta=(1+\lambda h)$.

We can see that the maximum spacing error is defined by $L_{e}$ and $a_{\min }$ the maximum deceleration only, regardless of the speed of platoon.

\section{Follower Hard Braking}

The safety of the platoon in leader's hard braking scenario is not sufficient to ensure platoon safety. One important scenario to discuss is a hard braking of a vehicle other than the leader. In this scenario, the $n$th vehicle applies a hard braking $W_{n}=a_{n}=a_{m i n}$. It no longer follows the platoon control law, so it leaves the platoon. This may introduce a big problem concerning the other vehicles moving behind the decelerating vehicle. Safety requirements impose to change the control laws of the follower vehicles and to stop following the original platoon. So the original platoon will be divided into two sub platoons. The first platoon consists of the vehicles ahead of the decelerating vehicles; this platoon will keep moving without any changes (assuming that the shared speed $V$ is equal to $v_{L}$ ) and without any effects on stability and safety. The second platoon consists of the decelerating vehicle and all the vehicles behind it. The decelerating vehicle becomes the leader of the new platoon. The control law for the followers is the same as in (3), except that the shared speed $V$ becomes, equal to the speed of the new leader $v_{n}$ :

$$
W_{j}=\frac{1}{h} \dot{e}_{j}+\frac{\lambda}{h} e_{j}-\lambda\left(v_{j}-v_{n}\right), \quad j>n
$$

From the previous control law we can see that we can apply all the discussion done before. We get the same error dynamics, hence the same spacing transfer function. This gives us the same stability and safety conditions. So in follower hard braking scenario, we get two stable and safe platoons without any additional conditions. The only thing to do is to divide the original platoon into two sub platoons and the first decelerating vehicle becomes the leader of the second platoon.

\section{Safety With Communication Loss}

As discussed before, each vehicle decreases the value of $V$ separately when a communication loss is detected. To maintain stability the decrease rate must be the same for all vehicles. The rate is imposed by safety requirements in the worst communication loss scenario. The worst scenario is to lose communication during hard braking.

In the hard braking scenario with full communication, the rate of deceleration of $V$ is equal to $a_{\min }$ because $V=v_{L}$. We also choose this rate $\left(a_{m i n}\right)$ as decrease rate for $V$ in case of communication loss. This gives the platoon a similar response in hard braking with and without communication. So we get a smooth response and we maintain platoon safety without any additional safety conditions.

Losing communication is more critical in follower braking scenario. In this scenario, the vehicles behind the decelerating vehicle must be informed to stop following the original leader and to follow the new leader. No problem appears if communication is not lost. On the other hand, the decelerating vehicle may not be able to inform all the vehicles in case of communication loss. But this is not critical because all vehicles automatically switch to autonomous mode. Each vehicle begins decreasing the value of $V$ with the maximum deceleration rate. So the vehicles behind the decelerating vehicle react exactly as if there were a communication ( $V$ decreasing at the same rate as $v_{n}$ ) even without knowing about the hard braking. So the platoon is still safe without any additional conditions. The hard braking of the leader in the event of communication loss is a special case of the previous scenario.

Decreasing $V$ at $a_{\min }$ rate makes $V$ reach zero rapidly. This may cause discomfort to the passengers after each communication loss. To decrease this bad effect we can change the value of control parameters $h, \lambda$ in such a way that we get comfortable response without losing safety and stability.

\section{E. Communication Loss Manoeuvre Delay}

Inter-vehicle communication loss cannot be detected instantaneously. It usually takes some time $\delta t$ to detect and to inform all the vehicles about the event. This interval has a critical effect on platoon safety. $\delta t$ can be fixed and well determined so that all vehicles in the platoon are simultaneously aware of communication loss. In the following discussion we give a method to check the safety of the platoon in the worst communication loss scenario, taking $\delta t$ into account. Other communication delays and clock synchronization between vehicles are not discussed.

In this case the worst communication loss scenario is to lose communication at $t_{h}$ when moving at maximum speed and at the same time the leader applies hard braking. This scenario is divided into three stages shown in fig. 3 : 
- Stage 1 begins at $t_{h}$, when the leader applies the hard braking and at the same time communication is lost. The leader decelerates at the maximum deceleration rate. The value $V$ is not updated any more and remains equal to the last broadcasted value. The worst case is to assume that the last received speed is $V=v_{\max }$, and all the follower vehicles are moving at speed $v_{\max }$.

- Stage 2 begins at $t_{c}$ when all vehicles are informed about communication loss. They switch to full autonomous mode by decreasing $V$ at maximum deceleration rate.

- Stage 3 begins when the leader stops at $t_{s_{L}}$, until the first follower stops at $t_{s_{1}}$.

To check the safety of the platoon we have to study the final value of the spacing between the leader and the first follower $\Delta\left(t_{s_{1}}\right)=x_{L}\left(t_{s_{1}}\right)-x_{1}\left(t_{s_{1}}\right)$. It must be greater than zero, otherwise a collision takes place.

To calculate $\Delta\left(t_{s_{1}}\right)$ we assume the worst case in the first stage, then at the second and third stages we have to find the dynamics of first spacing error. This enables us to calculate the first error and its derivative at $t_{s_{1}}$. The dynamics of the first error given in (8) cannot be used in this scenario because $V$ is not equal to $v_{L}$ any more. The new dynamics can be calculated using (3), and by adding and subtracting $h a_{L}, \lambda v_{L}$ we obtain:

$$
h \ddot{e}_{1}+(1+\lambda h) \dot{e}_{1}+\lambda e_{1}=h a_{L}+\lambda h\left(v_{L}-V\right)
$$

During the second stage we have $a_{L}=a_{m i n}$, $V=v_{\max }+a_{\min } t$ and $v_{L}=v_{\max }+a_{\min }(\delta t+t)$. So we get :

$$
h \ddot{e}_{1}+(1+\lambda h) \dot{e}_{1}+\lambda e_{1}=h a_{\text {min }}+\lambda h a_{\text {min }} \delta t
$$

We find the solution of the previous differential equation with the initial values at $t_{c}$ given in fig. 3 and we get $e_{1}(t)$ and $\dot{e}_{1}(t)$ in the interval $t \in\left[t_{c}, t_{s_{L}}\right]$ :

$$
\begin{gathered}
e_{1}(t)=\alpha_{1} \exp \frac{-\left(t-t_{c}\right)}{h}+\alpha_{2} \exp ^{-\lambda\left(t-t_{c}\right)}+\alpha_{3} \\
\dot{e}_{1}(t)=\frac{h a_{\text {min }}(1+\lambda \delta t)}{(\lambda h-1)} \exp \frac{-\left(t-t_{c}\right)}{h}-\frac{a_{\min }(h+\delta t)}{(\lambda h-1)} \exp ^{-\lambda\left(t-t_{c}\right)}
\end{gathered}
$$

where

$$
\begin{aligned}
& \alpha_{1}=-\frac{h\left(2 a_{\min } \delta t(\lambda h-1)+2 L e \lambda+a_{\min }\left(2 h-\lambda \delta t^{2}\right)\right)}{2(\lambda h-1)} \\
& \alpha_{2}=\frac{2 L e \lambda+a_{\min }\left(2 h-\lambda \delta t^{2}\right)}{\lambda(\lambda h-1)}, \alpha_{3}=\frac{h a_{\min }(1+\lambda \delta t)}{\lambda}
\end{aligned}
$$

The second stage ends at $t_{s_{L}}$ when the leader stops, so its duration is $\delta t_{1}=-\frac{v_{\max }}{a_{\min }}$. We calculate the initial values $x_{1}\left(t_{s_{L}}\right), v_{1}\left(t_{s_{L}}\right)$ and $e_{1}\left(t_{s_{L}}\right)$ for the third stage using (19) and (18) at $t_{s_{L}}$. During the third stage we have $v_{L}=0$ and $a_{L}=0$ so from (15) we get the dynamics of $x_{1}$ :

$$
h \ddot{x}_{1}+(1+\lambda h) \dot{x}_{1}+\lambda x_{1}=\lambda h a_{\min } t+h x_{L}\left(t_{s_{L}}\right)
$$

Solving the differential equation with the initial values at $t_{s_{L}}$ we get $x_{1}(t)$ and $v_{1}(t)$ :

$$
x_{1}(t)=\beta_{1} \exp \frac{-\left(t-t_{s_{L}}\right)}{h}+\beta_{2} \exp ^{-\lambda\left(t-t_{s_{L}}\right)}+\beta_{3}\left(t-t_{s_{L}}\right)+\beta_{4}
$$

$$
\begin{aligned}
& t \in\left[t_{s_{L}}, t_{s_{1}}\right] \\
& \dot{v}_{1}(t)=\gamma_{1} \exp ^{-\lambda\left(t-t_{s_{L}}\right)}+\gamma_{2} \exp \frac{-\left(t-t_{s_{L}}\right)}{h}+h a_{m i n}
\end{aligned}
$$

where:

$$
\begin{aligned}
& \beta_{1}=\frac{h\left(v_{1}\left(t_{s_{L}}\right)+x_{1}\left(t_{s_{L}}\right) \lambda-h x_{L}\left(t_{s_{L}}\right)+\lambda h^{2} a_{m i n}\right)}{\lambda h-1} \\
& \beta_{2}=\frac{\left(x_{1}\left(t_{s_{L}}\right) \lambda+\lambda v_{1}\left(t_{s_{L}}\right) h-h x_{L}\left(t_{s_{L}}\right)+h a_{m i n}\right)}{(\lambda h-1) \lambda} \\
& \beta_{3}=h a_{m i n}, \beta_{4}=\frac{\left(-h a_{m i n} \lambda+x_{L}\left(t_{s_{L}}\right)-a_{m i n}\right) h}{\lambda} \\
& \gamma_{1}=\frac{-v_{1}\left(t_{s_{L}}\right)}{\lambda h-1}, \gamma_{2}=-\frac{h\left(-\lambda v_{1}\left(t_{s_{L}}\right)+a_{\min }(\lambda h-1)\right)}{\lambda h-1}
\end{aligned}
$$

From (22) we calculate the time needed for the first vehicle to stop $\left(t_{s_{1}}-t_{s_{L}}\right)$. Then we can compute the final value of $x_{1}$. To check the safety we check if $x_{L}\left(t_{s_{1}}\right)-x_{1}\left(t_{s_{1}}\right)>0$.

The previous calculation can be performed for a range of values of $\delta t$ and we draw the spacing as a function of $\delta t$. The value of $\delta t$ at which the final spacing becomes equal to zero will be the maximum value of $\delta t$ that keeps the platoon safe.

\section{Simulations}

Simulations have been performed to check the effectiveness of the proposed control and its safety in the previous proposed scenarios. We used Matlab to check the control law using the ideal system model given in (1). We have also used The Open Racing Car Simulator (TORCS) to verify the safety of the platoon with a more realistic model. The results given in the paper are those obtained with TORCS except the first simulation which was done in Matlab. TORCS is one of the most popular car racing simulators for academic purposes. It features a sophisticated physics engine (aerodynamics, fuel consumption, traction...) as well as a 3D graphics engine for the visualization of the races.

Simulation were performed using a straight track. A platoon of 10 identical cars move along this track. The desired intervehicle distance is $5 \mathrm{~m}$, the maximum speed in normal mode $v_{\max }=140 \mathrm{~km} / \mathrm{h}$ and maximum deceleration $a_{\text {min }}=5 \mathrm{~m} / \mathrm{s}^{2}$. Control parameters are $h=1.5$ and $\lambda=3$. These parameters give a maximum control error $L_{e}=2.5 \mathrm{~m}$.

To check safety we test the following scenarios:

1) Applying hard braking when the platoon is moving at very high speed, to check the maximum limit of the error.

2) Leader hard braking with full communication,

3) Follower hard braking with full communication,

4) Leader hard braking with total communication loss,

5) Leader hard braking with total communication loss and with large interval $\delta t$.

In fig. 4 the platoon accelerates with maximum acceleration until it reaches very high speed $(250 \mathrm{~km} / \mathrm{h})$ then maximum hard braking is applied. This simulation is done in Matlab, just to show that the maximum error will saturate at a limit $L_{e}$ defined by $a_{\min }$ regardless of the maximum speed. It is obvious that the errors saturate at this maximum error 


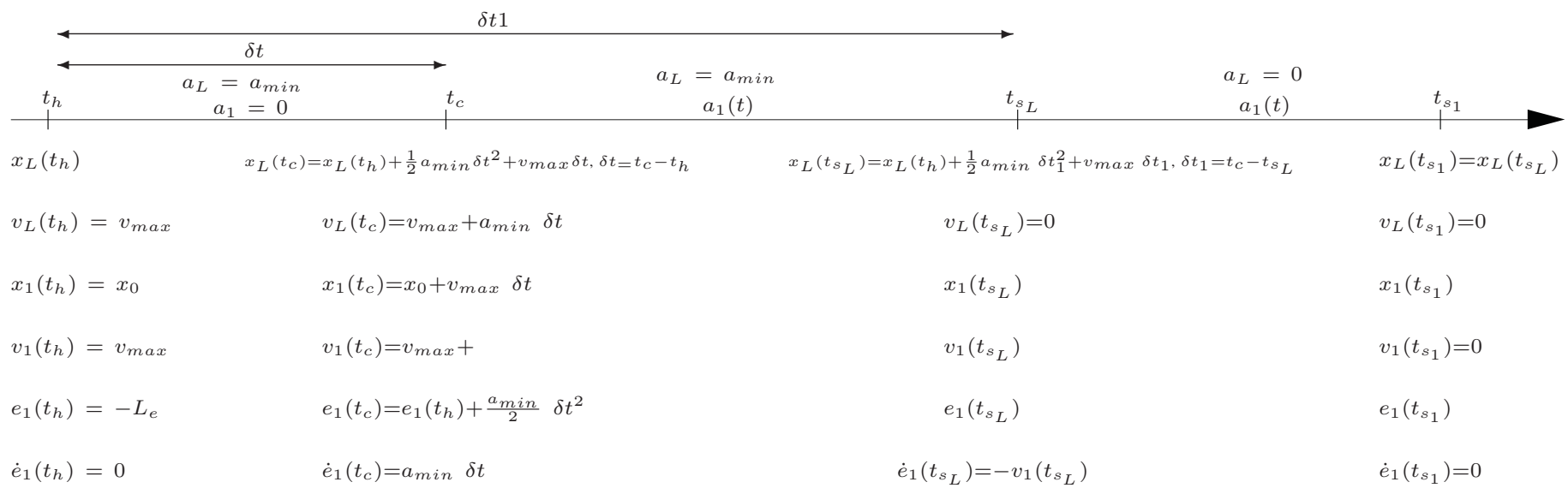

Fig. 3. Stages of worst communication loss scenario with initial conditions
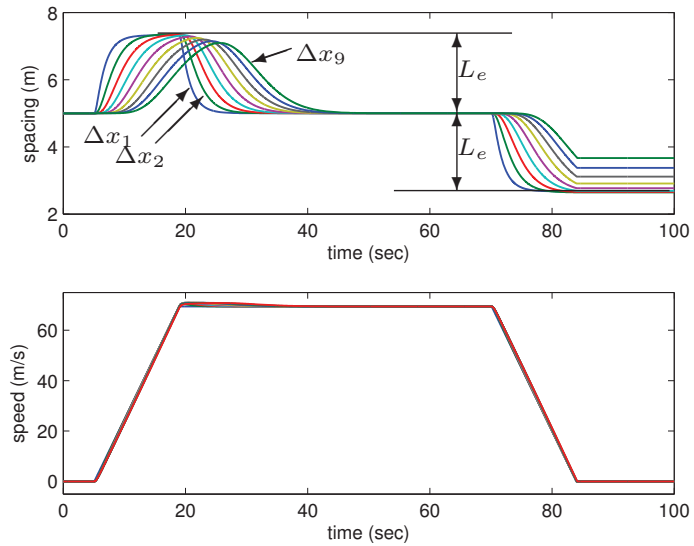

Fig. 4. Maximum spacing error limit

limit $L_{e}$, although the vehicles are still accelerating. Then when the vehicles reach the maximum speed and they stop accelerating, the spacing errors decrease toward zero. In hard braking scenario, the errors also do not exceed $L_{e}$. But in this case, the errors do not converge again toward zero because the vehicles are stopped. So no collision will take place even at very high speeds.

In all the following experiments, the platoon moves at the maximum allowed speed when we apply a hard braking according to each scenario.

In the second scenario, the leader applies hard braking with full communication. Fig. 5 shows the results. It is clear that the platoon is safe, since the spacing is always greater than zero.

In the follower hard braking scenario shown in fig. 6 , the fifth vehicle applies a hard braking. The platoon is divided into two platoons. The first platoon continues to move safely at the same speed without any change in the spacing and speed. The second platoon decelerates until it stops without any collision. Notice that $\Delta_{x_{5}}$, increases since the fifth vehicle stops and the fourth vehicle is still moving.

In fig. 7 the leader applies a hard braking and, at the same time, communication between vehicles is totally lost.
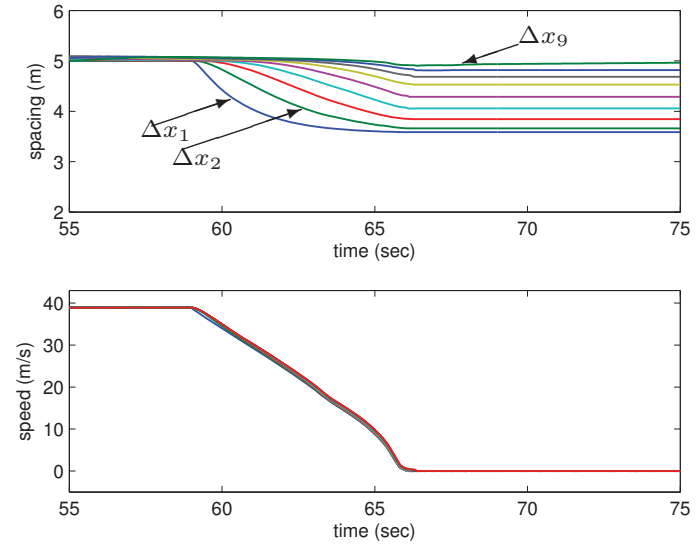

Fig. 5. Leader hard braking with full communication
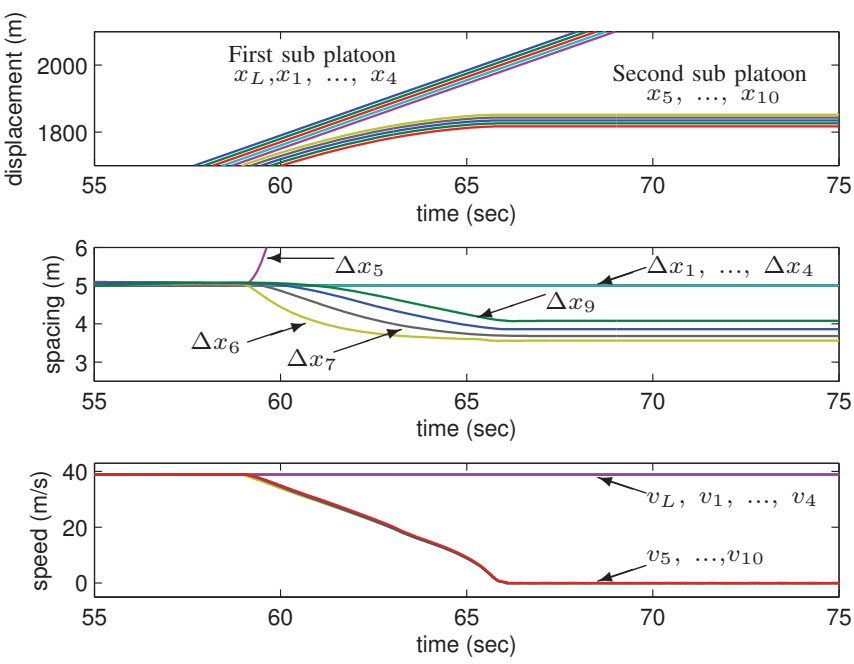

Fig. 6. Spacing and speed of first and second sub platoons, during follower hard braking with full communication 

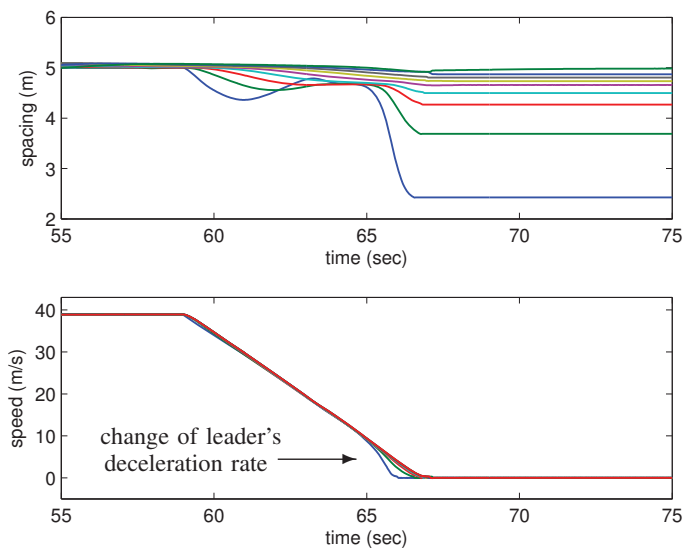

Fig. 7. Leader hard braking with no communication
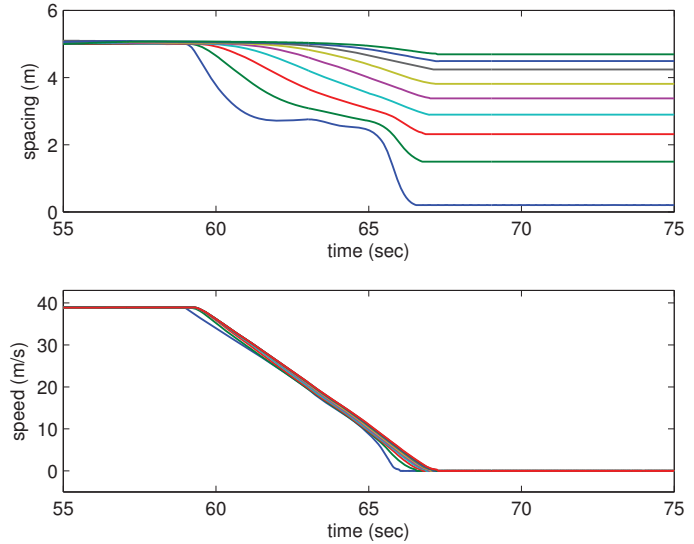

Fig. 8. Leader hard braking and communication loss with maximum allowed delay to inform all vehicles about communication loss

We can see that the platoon is still stable and safe. We can see a non linear change in spacing just before stopping. This non linearity is due to non perfect linearization of the car in TORCS, especially when changing the gears. It is obvious that the leader changes its deceleration rate before stopping, so the amplitude of the spacing error changes accordingly.

In the last experiment we check the maximum allowed delay interval to inform all the vehicles in the platoon about communication loss. We perform the computation of the spacing for a range of delay values. Then we draw the spacing as a function of $\delta t$. The value of $\delta t$ for which the spacing becomes equal to zero is the maximum bound for the delay. In our case we found $\delta t \approx 0,35$. To check the validity of this value we run a simulation with a large delay $\delta t=0,3$. In this case the spacing becomes very small but the platoon is still safe, as shown in fig. 8.

\section{CONCLUSION}

We have addressed the safety of platooning on highways when using the flatbed tow truck model and the modified $\mathrm{CTH}$. We found safety conditions of the platoon and proved the safety of the platoon in the most critical hard braking scenarios with and without communication loss. The effect of the delay to inform all vehicles about communication loss has also been studied. A method has been proposed to find the maximum delay without losing safety. Simulations have been performed with 10 vehicles to check the safety in the proposed scenarios.

The effect of lags and communication delays must be studied in the near future to verify the robustness and the safety of the control in even more realistic scenarios. In addition, passenger comfort must also be taken into account.

\section{REFERENCES}

[1] A. Ali, G. Garcia, and P. Martinet, Minimizing the inter-vehicle distances of the time headway policy for platoons control in highways, 10th International Conference on Informatics in Control, Automation and Robotics (ICINCO13), pp. 417-424. SciTePress, Reykjavik, Iceland, July 29-31, 2013.

[2] A. Ali, G. Garcia, and P. Martinet, Minimizing the inter-vehicle distances of the time headway policy for urban platoon control with decoupled longitudinal and lateral control, 16th International IEEE Conference on Intelligent Transportation Systems - (ITSC), pp. 1805-1810, The Hague, The Netherlands, 6-9 Oct. 2013.

[3] A. Ali, G. Garcia, and P. Martinet, The flatbed platoon towing model for safe and dense platooning on highways, IEEE Intelligent Transportation Magazine, to be published 2014.

[4] P. Li, L. Alvarez, R. Horowitz, AHS safe control laws for platoon leaders, IEEE Transactions on Control Systems Technology, vol.5, no.6, pp.614,628, Nov. 1997.

[5] L. Alvarez, R. Horowitz, Safe Platooning in Automated Highway Systems Part I: Safety Regions Design, Vehicle System Dynamics, Vol. 32, Iss. 1, 1999.

[6] L. Alvarez, R. Horowitz, Safe Platooning in Automated Highway Systems Part II: Velocity Tracking Controller, Vehicle System Dynamics: International Journal of Vehicle Mechanics and Mobility, 32(1):57-84, 1999.

[7] A. Ferrara, and C. Vecchio, Collision avoidance strategies and coordinated control of passenger vehicles, Nonlinear Dynamics, 49(4):475-492, 2007.

[8] G. Franck, C. Vincent and C. Francois, A Reactive Multi-Agent System for Localisation and Tracking in Mobile Robotics, ictai, pp. 431-435, 16th IEEE International Conf. Tools with Artificial Intelligence (ICTAI'04), 2004.

[9] P. Ioannou and C. Chien, Autonomous intelligent cruise control, IEEE Trans. Veh. Technol., 42(4):657-672, 1993.

[10] A. Peter Cook, Stable Control of Vehicle Convoys for Safety and Comfort, IEEE Transactions on Automatic Control, vol.52, no.3, pp. 526,531, March 2007.

[11] R. Rajamani, Vehicle dynamics and control. Springer Science, 1st ed.: Springer US, 2006.

[12] Ricardo (2009), Cars that drive themselves can become reality within ten years, [Online]. Available: http://www.ricardo.com/en-GB/NewsMedia/Press-releases/News-releases 1/2009/Cars-that-drive-themselvescan-become-reality-within-ten-years/

[13] A. Scheuer, O. Simonin, F. Charpillet, Safe longitudinal platoons of vehicles without communication, IEEE International Conference on Robotics and Automation (ICRA 09), pp.70,75, 12-17 May 2009. doi: 10.1109/ROBOT.2009.5152629

[14] D. Swaroop, String stability of interconnected systems: An application to platooning in automated highway systems, UC Berkeley: California Partners for Advanced Transit and Highways (PATH), 1997.

[15] D. Swaroop and K. Rajagopal, A review of constant time headway policy for automatic vehicle following, Proc. of IEEE Intell. Transp. Syst., pp. 6569, 2001.

[16] L. Xiao and F. Gao, Practical string stability of platoon of adaptive cruise control vehicles, IEEE Trans. Intell. Transp. Syst., 12(4):1184-1194, 2011.

[17] D. Yanakiev and I. Kanellakopoulos, A simplified framework for string stability analysis in AHS, Proc. 13th IFAC World Congress, pp. 177-182, 1996. 